\title{
Infrared spectrum and proper motion of the brown dwarf companion of HR 7329 in Tucanae*
}

\author{
E. W. Guenther ${ }^{1}$, R. Neuhäuser ${ }^{2}$, N. Huélamo ${ }^{2}$, W. Brandner ${ }^{3}$, and J. Alves ${ }^{4}$ \\ 1 Thüringer Landessternwarte Tautenburg, Sternwarte 5, 07778 Tautenburg, Germany \\ 2 MPI für extraterrestrische Physik, 85740 Garching, Germany \\ 3 University of Hawaii, Institute for Astronomy, 2680 Woodlawn Dr., Honolulu, HI 96822, USA \\ ${ }^{4}$ European Southern Observatory, Karl-Schwarzschild-Straße 2, 85748 Garching, Germany
}

Received 4 August 2000 / Accepted 24 October 2000

\begin{abstract}
Up to now only four brown dwarf companions to normal stars have been found and confirmed by both spectroscopy and proper motion (namely Gl 229 B, G 196-3 B, Gl 570 D, and CoD-33 7795 B). On the basis of an optical spectrum taken with HST/STIS Lowrance et al. (2000) recently pointed out another possible candidate companion. The companion candidate is located at a distance of $4^{\prime \prime}$ from the A0-star HR 7329, which is considered as a member of a moving group of young stars in Tucanae located at a distance of only $\sim 48$ pc. In order to confirm or disregard the companion nature of the candidate, we have determined the proper motion of the brown dwarf candidate with an epoch difference of 1.8 years, and found that it is consistent with a co-moving companion of HR 7329. Additional to the proper motion measurement, we have also taken an $H$-band spectrum using ISAAC on the ESO-VLT. From this spectrum, we conclude that the companion candidate has spectral type M 7 to M 8 , which is in agreement with the optical spectrum. We thus conclude that HR 7329 B is most likely a brown dwarf companion. The mass ratio of this pair (A0 to M 7-8, i.e. 100:1) is the largest known among brown dwarf companions, which is relevant for studying the formation of brown dwarfs as companions.
\end{abstract}

Key words. stars: binaries: visual - individual: HR 7329 - late-type - pre-main sequence

\section{Introduction}

Brown dwarfs are objects with masses below the stellar limit but with masses above planets. They thus form a natural bridge between low-mass stars and planets. Brown dwarfs are unable to sustain stable nuclear fusion of hydrogen, but can burn deuterium until they are $\sim 10^{7}$ yrs old. The sub-stellar limit, i.e. the dividing line between stars and brown dwarfs is the hydrogen burning mass, namely $\sim 0.078 M_{\odot}$, depending on metallicity. Planets cannot even burn deuterium and have masses below $\sim 0.01 M_{\odot}$. However, the important physical difference between brown dwarfs and planets seems to be the way in which they form. One apparent difference between planets and brown dwarfs is that the brown dwarfs are unlikely to be found as close companions of stars (Latham et al. 1989; Mayor \& Queloz 1995; Marcy \& Butler 1998; Zucker \& Mazeh 2000). This is often referred to as the brown-dwarf desert. In contrast to this, numerous free-floating brown

Send offprint requests to: E. Guenther,

e-mail: guenther@tls-tautenburg.de

* Based on observations obtained at the European Southern Observatory on Cerro Paranal and La Silla in program 65.L-0144. dwarfs have been discovered by direct imaging searches. Interestingly, some of the free-floating objects seem to have masses below the deuterium burning limit (Zapatero Osorio et al. 2000a; Zapatero Osorio et al. 2000b). Since the masses of these objects seem to be of the order of $\sim 0.01 M_{\odot}$ or even lower, they are often referred to as freefloating planets. On the other hand, they could also be considered as extremely low-mass brown dwarfs. The dividing line between brown dwarfs and planets is currently controversial and poorly understood. The fact that planets in orbit around solar-mass stars exist, whereas brown dwarfs are at least extremely rare, must apparently be a consequence of the way in which these objects form. In order to shed more light on to this problem, it is necessary to find out how empty this brown-dwarf companion desert really is, and what the properties of the brown-dwarfs that are found in orbit around a star are. Can stars of all masses have brown dwarf companions? Do isolated and companion brown dwarfs have the same mass function, or does the mass function depend of the companion on the mass of the primary? Are the orbital-characteristics of the browndwarf companions different from those of planets? 
Up to now, only very few brown dwarfs were found as companions to normal stars: Gl $229 \mathrm{~B}$ (Nakajima et al. 1995; Oppenheimer et al. 1995), G 196-3 B (Rebolo et al. 1998), Gl570D (Burgasser et al. 2000), and CoD-337 795 B (suggested as sub-stellar companion candidate by Lowrance et al. 2000, and confirmed as such by Neuhäuser et al. 2000b), all of which are brown dwarfs confirmed as companions by both spectroscopy and proper motion. Two more candidates were suggested, namely GG Tau Bb (White et al. 1999) and HR 7329 B (Lowrance et al. 2000, henceforth L2K), the latter is investigated in this paper. In those two companion candidates, optical spectra are taken, but proper motion was not yet available. Brown dwarfs and L-dwarfs can have brown dwarf companions themselves (Basri \& Martín 1999; Martín et al. 1999a). In view of the fact that brown dwarf companions are so rare, it is advisable to ensure that these companions are really brown dwarfs. Thus not only the spectral types have to be determined, but it also has to be shown that the object is orbiting the primary (or at least co-moving with it in case the orbital period is too long). There is no direct imaging detection of an extra-solar planet, yet. Previous candidates for ejected (Terebey et al. 1998), or orbiting (Neuhäuser et al. 2000a) planets could not be confirmed as cool planets by spectroscopy (Terebey et al. 2000; Neuhäuser et al. 2000c).

Like L2K, we also search for substellar companions to young nearby stars, where companions are still relatively bright and well-separated (see Neuhäuser et al. 2000a, 2000b). We use ground-based facilities, mainly the NTT on La Silla, the VLT on Cerro Paranal, and ALFA, the adaptive optics infrared imaging instrument on the Calar Alto $3.5 \mathrm{~m}$.

By investigating archived HST NICMOS data, we also noticed the faint object near the presumably young star HR 7329 (Sect. 2) and then performed follow-up infrared spectroscopy, which we present in Sect. 3. By comparing the relative positions of HR $7329 \mathrm{~A}$ and B in our new acquisition image with the two year old archived HST NICMOS image (published by L2K), we estimate the proper motion of HR 7329 B (Sect. 4). We discuss our findings in Sect. 5.

\section{HR 7329 A and B in Tucanae}

Simultaneously and independently, both Zuckerman \& Webb (2000) and Torres et al. (2000) presented evidence for two nearby young moving groups called Tucanae cluster and Horologium association, respectively. The probable and likely members of the Tuc and Hor A groups are both at $\sim 40$ to $50 \mathrm{pc}$ (mostly measured by the Hipparcos satellite) with signatures for youth in their spectra like $\mathrm{H} \alpha$ emission and Lithium absorption lines. They show similar radial velocities and proper motions and are located close to each other. Hence, both groups may very well form one large moving group altogether. The young star $\iota$ Hor is probably also located in HorA (Torres et al. 2000). Recently, Kürster et al. (2000) found radial velocity variations of this star that are consistent with the presence of a planet, and it may also have a circumstellar disk.

By comparing the X-ray luminosity function of the Tuc members observed by ROSAT with those of the $\mathrm{T}$ Tauri stars in the Taurus and TW Hya associations as well as with zero-age main-sequence stars of the Pleiades, Stelzer \& Neuhäuser (2000) concluded that the Tuc stars have an age of 10 to 30 Myrs, i.e. young, but close to or already on the zero-age main-sequence.

The star HR 7329 is listed as probable member of the Tucanae group (Zuckerman \& Webb 2000). It has a spectral type A0 and its radial velocity and proper motion are consistent with kinematic membership. Most recently, L2K presented evidence for a brown dwarf companion (called HR 7329 B), located $4^{\prime \prime}$ off the star HR 7329 A. The companion candidate HR $7329 \mathrm{~B}$ was found to have a spectral type M 7 to M 8 in their HST/STIS spectrum.

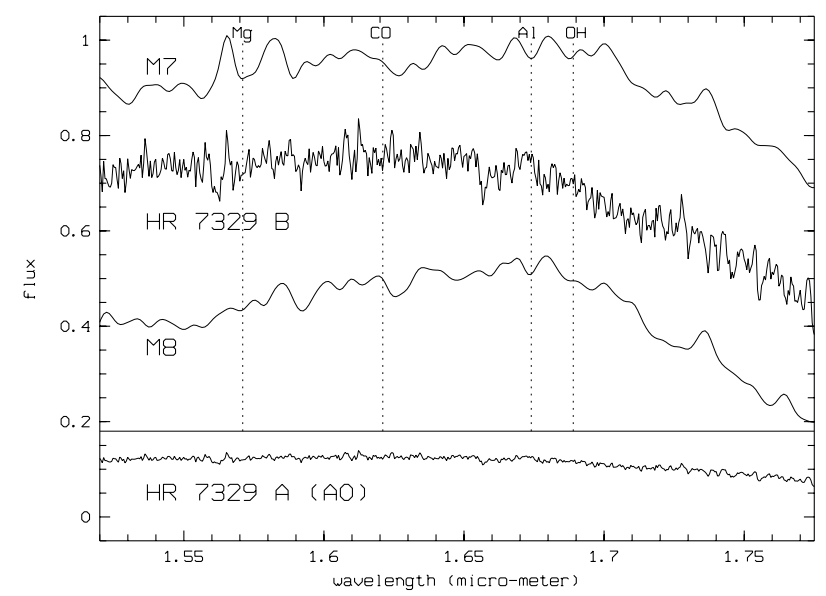

Fig. 1. Our $H$-band spectrum of HR 7329 B compared with the young $\mathrm{M} 7$ (average of $\mathrm{Cha} \mathrm{H} \alpha 2,3,6$, and 8) and $\mathrm{M} 8$ dwarfs (average of $\mathrm{Cha} \mathrm{H} \alpha 1,7,10$, and 11) showing that our object has spectral type M 7 to $M$ 8. The comparison spectra are taken from Comerón, et al. (2000). Also plotted is our $H$ band spectrum of HR 7329 A.

To confirm a companion candidate found by direct imaging as true companion, one needs to show not only that its spectral type is consistent with its colours and with the observed magnitude difference (assuming the same distance), but also that it is co-moving with its primary star. Orbital motion would be a final proof of companionship.

\section{The $H$-band spectrum of HR 7329 B}

We obtained an $H$-band spectrum $(R \simeq 500)$ on 16 th of April 2000 with the Infrared Spectrograph and Array Camera (ISAAC) at the European Southern Observatory (ESO) $8.2 \mathrm{~m}$ telescope Antu, Unit Telescope No. 1 (UT1) of the Very Large Telescope (VLT). The spectrum consists of 20 co-added $60 \mathrm{~s}$ exposures through a $0.6^{\prime \prime}$ slit, aligned neither along the position angle of the pair nor perpendicular to it, but in between those two positions, so that 
the two objects are well separated and that the flux from the companion candidate is several times larger than the flux from the bright star. Darks, flats, and standards were taken in the same night.

After standard data reduction, we modeled and subtracted the flux of the bright star from the faint object's spectrum at each wavelength. The final spectra of both HR 7329 A and B are shown in Fig. 1. The primary and HD 188112 (van der Bliek et al. 1996) were used to obtain a relative flux-calibration. For very late-type objects, the shape of the continuum in the near-infrared is sensitive to the spectral type (Kirkpatrick 2000). Comparing our spectrum with spectra of young M 7 and M 8-type dwarfs from the Cha I dark cloud (Comerón et al. 2000), we find that HR $7329 \mathrm{~B}$ has spectral type M 7 to M 8. As pointed out by Martín et al. (1999a) the $\mathrm{H}_{2} \mathrm{O} H$-band index at $1.51-1.57 \mu \mathrm{m}$ can also be used to determine the spectral types of very late $M$, and L-stars. Using this index, we also derive a spectral type of M 7 to M 8 for HR $7329 \mathrm{~B}$. These results agree well with the optical spectrum taken with HST/STIS, presented by L2K.

\section{Positional measurements of HR 7329 B}

HR 7329 B was first detected by L99 using HST NICMOS on 29 June 1998 in the F160W filter. The object was then located $0.95 \pm 0.04^{\prime \prime}$ east and $4.06 \pm 0.04^{\prime \prime}$ south of HR $7329 \mathrm{~A}$, corresponding to a separation of $\rho=4.17 \pm$ $0.05^{\prime \prime}$ and a position angle of $\theta=168.8 \pm 0.2^{\circ}$ measured from north over east to south.

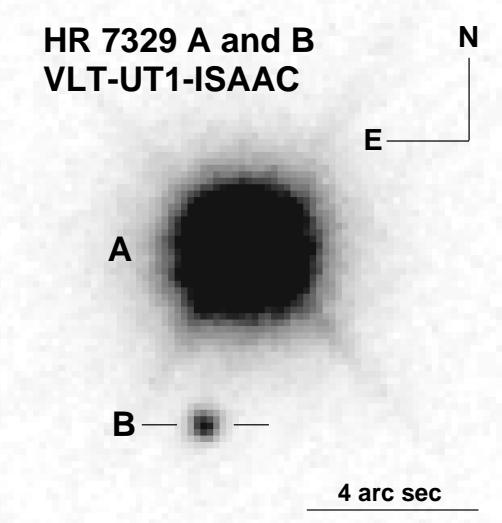

Fig. 2. ISAAC acquisition image of HR $7329 \mathrm{~A}$ and $\mathrm{B}$, the FWHM is $0.48^{\prime \prime}$

We detected HR 7329 B in our $2 \mathrm{~s}$ exposure ISAAC $\left(0.147^{\prime \prime} / \mathrm{pixel}\right)$ acquisition image taken on the $22 \mathrm{nd}$ of April 2000 using two narrow band filters centered on $2.17 \mu \mathrm{m}(\mathrm{Br} \gamma$ ) and $2.19 \mu \mathrm{m}$ (with $\Delta \lambda=0.028$ and $0.03 \mu \mathrm{m}$, respectively). The seeing was $\sim 0.5^{\prime \prime}$, and both objects are clearly detected and resolved. Star A is not saturated. The magnitude difference between star A and object $\mathrm{B}$ in the acquisition image (Fig. 2) is 5.7 mag.
Table 1. Proper motion of HR 7329

\begin{tabular}{llll}
\hline Catalogue & $\begin{array}{l}\text { designation } \\
\text { in catalogue }\end{array}$ & $\begin{array}{l}\mu_{\alpha} \\
{\left[\mathrm{mas} \mathrm{yr}^{-1}\right]}\end{array}$ & $\begin{array}{l}\mu_{\delta} \\
{\left[\mathrm{mas} \mathrm{yr}^{-1}\right]}\end{array}$ \\
\hline Hipparcos & HIP 95261 & $25.57 \pm 0.75$ & $-83.03 \pm 0.49$ \\
PPM & PPM 347630 & $29.7 \pm 3.5$ & $-82.0 \pm 3.8$ \\
TRC & TRC 8765-2571 & $25.7 \pm 3.5$ & $-78.1 \pm 2.3$ \\
ACT & ACT 8765-2571 & $25.4 \pm 5.1$ & $-79.3 \pm 12.4$ \\
\hline
\end{tabular}

The companion candidate was located $0.93 \pm 0.04^{\prime \prime}$ east and $3.99 \pm 0.04^{\prime \prime}$ south of the bright star, corresponding to $\rho=4.097 \pm 0.048^{\prime \prime}$ and $\theta=166.90 \pm 0.42^{\circ}$, consistent with the NICMOS image (L2K).

Using the infrared imager SofI (Son of Isaac) at the ESO 3.5 m New Technology Telescope (NTT) on La Silla we took an additional image on which both HR $7329 \mathrm{~A}$ and $\mathrm{B}$ are detected, and resolved, despite $\sim 1.2^{\prime \prime}$ seeing conditions. This image was taken on 17th of May 2000 with the H-band filter using the so-called "small SofI field", to obtain the highest angular resolution possible $\left(0.147^{\prime \prime} / \mathrm{pixel}\right)$. The image was exposed for $10 \mathrm{~min}$ total $(460 \times 1.3 \mathrm{~s}$ in auto-jitter mode). Darks, flats, and standard stars were taken in the same night, and we performed standard data reduction using eclipse and MIDAS.

In the SofI image, HR 7329 B is located $1.09 \pm 0.47^{\prime \prime}$ east and $4.31 \pm 0.27^{\prime \prime}$ south of the bright star, corresponding to $\rho=4.45 \pm 0.54^{\prime \prime}$ and $\theta=165.8 \pm 6.7^{\circ}$. Although this is consistent with the NICMOS and ISAAC images, the precision is much lower, due to the very bad seeing. Hence, we disregard this image from further analysis. This is not a big loss of information, since the maximum epoch difference would not increase by much if we would include the SofI image.

\section{Discussion}

The offsets in right ascension and declination as well as the separations and position angles observed for the HR7329 A and B pair with HST/NICMOS and VLT/ISAAC as given above are consistent with each other within $1 \sigma$. However, this does not prove object $\mathrm{B}$ to be a companion of star A. Whether we can already show that the motion of HR $7329 \mathrm{~B}$ relative to star A is inconsistent with B being an unrelated field star, depends on the proper motion of HR $7329 \mathrm{~A}$.

L2K argued that HR $7329 \mathrm{~B}$ is possibly co-moving with A, as B was in the 0". $2 \mathrm{HST} / \mathrm{STIS}$ slit when they took the spectrum on 22nd of May 1999, using the offset determined from an image taken about one year earlier. Using all, except the SofI image, we can now investigate this point with much higher accuracy than before.

Table 1 gives proper motion of HR 7329 taken from four different catalogues. For the rest of the paper, we will use the Hipparcos data, because it has the highest accuracy.

Figure 3 shows the proper motion of the A and B component. The position of the A-component on the 29th of June 1998 is plotted at the origin $((\alpha, \delta)=(0,0))$, and the 


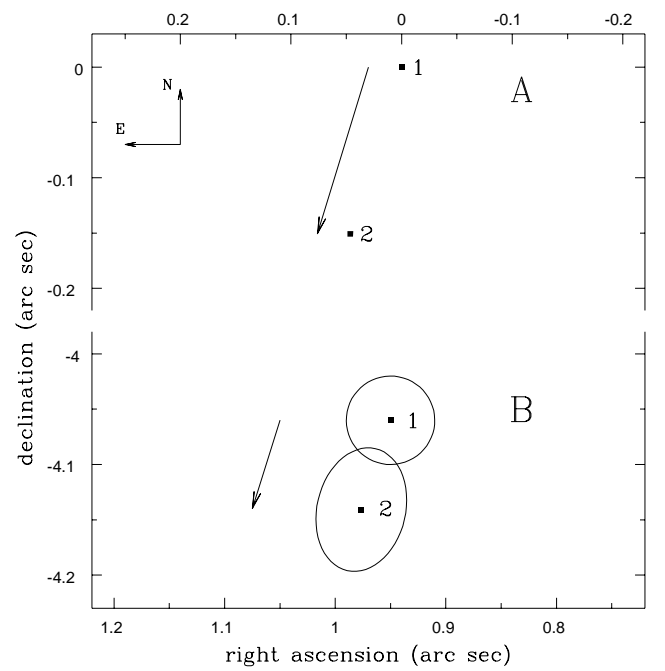

Fig. 3. Motion of companion candidate B and star A: Plotted are $\alpha$ and $\delta$ offset as given in the text and taking into account the known proper motion of star A, starting at the first epoch at $(\alpha, \delta)=(0,0)$, then moving to the south-west. Since the elipses slightly overlap the possibility of being an unrelated object cannot be completely excluded. However, since both objects seem to move in the same direction, it is far more likely that the objects are co-moving

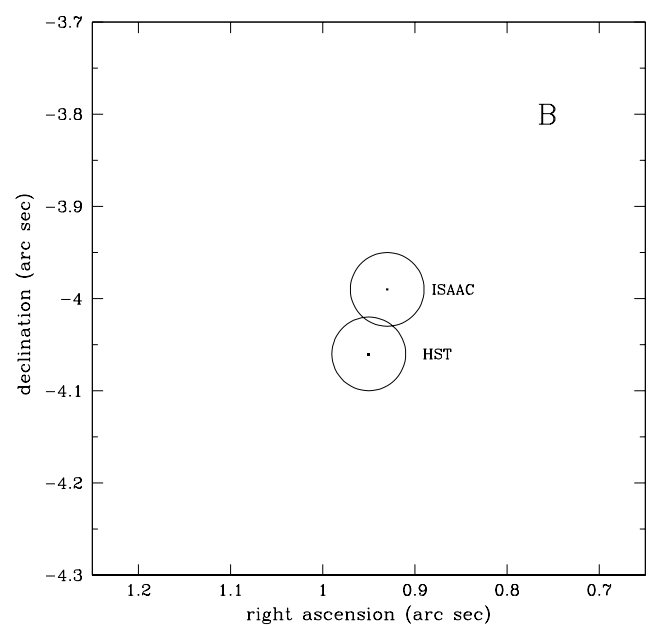

Fig. 4. Another way to investigate whether B is a non moving background object, or not is shown here. Plotted are just the two measured offsets (HST and ISAAC) of object B relative to star A, without taking any proper motion into account. If $\mathrm{A}$ and $\mathrm{B}$ form a common proper motion pair, then the error ellipses should overlap, which they do. If B is a background source that does not move, the error ellipses would be well separated, given the relatively large proper motion of the Acomponent. Using the known proper motion, we can estimate that the probably for that is only $\sim 0.5 \sigma$, only

position on the 22nd of April 2000 south-east of it is given by its proper motion. The errors in the position of star A at the 2nd epoch is given by the error of the Hipparcos proper motion. In addition, we plot the offsets of object $\mathrm{B}$ relative to star A with errors given by the errors of the measured offsets and the proper motion of star A. Clearly visible is that object $\mathrm{B}$ moves in to the same direction as $\mathrm{A}$. The amount of the proper motion of $B$ is within the errors, also consistent with the proper motion of A. However, the error-ellipses of the HST and the ISAAC observation do overlap. Thus formally, a non-moving background object would be possible. In order to test this hypothesis we plotted in Fig. 4 the two measured offsets (HST and ISAAC) of object $\mathrm{B}$ relative to star $\mathrm{A}$, without taking any proper motion into account. If $\mathrm{A}$ and $\mathrm{B}$ form a pair then the error ellipses should overlap (ignoring orbital motion), which they do. If the object were a non-moving background object, the position should within the errors remain the same in Fig. 3. While the errors are large, this is clearly not the case. While we cannot completely rule out that the companion candidate is a non-moving background object, it is far more convincing that it is co-moving. From Fig. 3 we conclude that the proper motions of A and B are similar, namely by $1 \sigma$ regarding their amount and by $2 \sigma$ regarding their direction. However, while this demonstrate that both $\mathrm{A}$ and $\mathrm{B}$ are members of a co-moving group of objects, it does not strictly demonstrate that B is orbiting around A. On the other hand, the probability for HR 7329 A and $\mathrm{B}$ to be two non-bound Tuc members being located that close together by chance, is very low.

As already discussed in L2K, the M 7 to M 8-type object HR $7329 \mathrm{~B}$ would be located at a distance of $\sim 19 \mathrm{pc}$, if it were a foreground main sequence dwarf. To find such a faint late-type object by chance $\sim 4^{\prime \prime}$ off HR 7329 is very unlikely, the probability being $\sim 10^{-7}$ (L2K).

If the $\mathrm{A}$ and B-components were at $48 \mathrm{pc}$ and $19 \mathrm{pc}$ respectively, the relative parallactic motion would be -32.11 mas in right ascension (towards the east) and -8.65 mas in declination (towards the south) for the HST and ISAAC observations. The corresponding error-circles would then have a slightly larger overlap than in Fig. 3, and the corresponding figure would be almost identical to Fig. 3. That object B is an unrelated, non-moving background object is extremely unlikely, because it would have to be very young given its magnitude and spectral type, if it would be located behind the Tucanae cluster. A nonmoving foreground object is also very unlikely. While a background object is strictly possible, it is less convincing than a co-moving object.

L2K derive an effective temperature of $2600 \pm 200 \mathrm{~K}$ and a bolometric luminosity of $0.0026 \pm 0.0003 L_{\odot}$ for HR $7329 \mathrm{~B}$ (at $48 \mathrm{pc}$ ) and compare its location in the HR diagram with th e evolutionary tracks and isochrones of Burrows et al. (1997) and Baraffe et al. (1998) to derive a mass range of $\sim 30$ to $50 M_{\text {jup }}$ at an age of $\sim 20$ to 30 Myrs. According to the new Chabrier et al. (2000) models, HR $7329 \mathrm{~B}$ has a mass of $\sim 20$ to $40 M_{\text {jup }}$. Given that the primary star HR $7329 \mathrm{~A}$ is an A0V star $\left(2.9 M_{\odot}\right)$, this is a binary with very high mass ratio $(\sim 0.01)$.

The angular separation of $4.15 \pm 0.05^{\prime \prime}$ corresponds to a projected physical separation of $200 \pm 10 \mathrm{AU}$ at $\sim 48 \mathrm{pc}$ distance. This in turn corresponds to an orbital period of $1660 \pm 130$ years and orbital motion of $15.7 \pm 1.4$ mas per year (for a face-on circular orbit), only a factor of 2 smaller 
than the relative astrometric precision achieved in the HST NICMOS and VLT ISAAC images discussed here. We thus conclude that HR 7329 B is most likely a brown dwarf companion of an $2.9 M_{\odot}$ star with a mass of only $\sim 20-50 M_{\text {jup }}$. The mass-ratio thus is the largest known among brown dwarf companions. As noted by Martín et al. (1999a) systems with very high mass ratios have large ( $\geq 100 \mathrm{AU})$ separations and systems with low mass ratios involving brown dwarfs have typically smaller separations $(\leq 20 \mathrm{AU})$. With a projected physical separation of $200 \pm 10$ AU HR 7329 seems to be another example of this rule.

Acknowledgements. We thank the referee, Gibor Basri, for his insightful comments on the paper. We would also like to thank the ESO User Support group with F. Comerón for assistance, the ISAAC team with J.-G. Cuby and C. Lidman for their service mode observations, the NTT team with L. Vanci, E. Le Floc'h, G. Martin, and J. Miranda for support during the NTT observations, and F. Comerón for providing his $H$ band spectra of late-type dwarfs in electronic form, as well as G. Wuchterl and S. Frink for very useful discussion. RN wishes to acknowledge financial support from the the Deutsches Zentrum für Luft- und Raumfahrt e.V. (DLR) under grant number 50 OR 0003.

\section{References}

Baraffe, I., Chabrier, G., Allard, F., \& Hauschildt, P. 1998, A\&A, 337, 403

Basri, G., \& Martín, E. L. 1999, AJ, 118, 2460

Burgasser, A. J., Kirkpatrick, J. D., Cutri, R. M., et al. 2000, ApJ, 531, L57

Burrows, A., Marley, M., Hubbard, W., et al. 1997, ApJ, 491, 856

Chabrier, G., Baraffe, I., Allard, \& F., Hauschildt, P. 2000, ApJ, in press [astro-ph/0005557]

Comerón, F., Neuhäuser, R., \& Kaas, A. A. 2000, A\&A, 359, 269

Kirkpatrick, J. D. 1998, Spectroscopic Properties of Ultracool Dwarfs and Brown Dwarfs, in Brown Dwarfs and Extrasolar Planets, ed. R. Rebolo, E. L. Martín, \& M. R. Zapatero Osorio, ASP Conf. Ser., 134, 405
Kürster, M., Endl, M., Els, S., et al. 2000, A\&A, 353, L33

Latham, D. W., Stefanik, R. P., Mazeh, T., Mayor, M., \& Burki, G. 1989, Nat, 339, 38

Lowrance, P. J., McCarthy, C., Becklin, E. E., et al. 1999, ApJ, 512, L69

Lowrance, P. J., Schneider, G., Kirkpatrick, J. D., et al. 2000, ApJ, 541, 390

Marcy, G. W., \& Butler, R. P. 1998, ARA\&A, 36, 57

Martín, E. L., Brandner, W., \& Basri, G. 1999, Sci, 283, 1718

Martín, E. L., Delfosse, X., Basri, G., et al. 1999, AJ, 118, 2466

Mayor, M., \& Queloz, D. 1995, Nat, 378, 355

Nakajima, T., Oppenheimer, B. R., Kulkarni, S. R., et al. 1995, Nat, 378, 463

Neuhäuser, R., Brandner, W., Eckart, A., et al. 2000a, A\&A, 354, L9

Neuhäuser, R., Guenther, E. W., Brandner, W., et al. 2000b, A\&A, 360, L39

Neuhäuser, R., Guenther, E. W., Brandner, W., et al. 2000c, Direct imaging search for planetary companions next to young nearby stars, in From darkness to light, ed. T. Montmerle, \& P. Andre, ASP Conf. Ser. [astro-ph/0007305]

Oppenheimer, B. R., Kulkarni, S. R., Matthews, K., \& van Kerkwijk, M. H. 1995, Sci, 270, 1478

Rebolo, R., Zapatero-Osorio, M. R., Madruga, S., et al. 1998, Sci, 282, 1309

Stelzer, B., \& Neuhäuser, R. 2000, A\&A, in press [astro-ph/0007350]

Terebey, S., van Buren, D., Padgett, D. L., Hancock, T., \& Brundage, M. 1998, ApJ, 507, L71

Terebey, S., van Buren, D., Matthews, K., \& Padgett, D. L. 2000, AJ, 119, 2341

Torres, C. A. O., Da Silva, L., Quast, G. R., de la Reza, R., \& Jilinski, E. 2000, AJ, 120, 1410

van der Bliek, N. S., Manfroid, J., \& Bouchet, P. 1996, A\&AS, 119,547

White, R. J., Ghez, A. M., Reid, I. N., \& Schulz, G. 1999, ApJ, 520,811

Zapatero Osorio, M. R., Béjar, V. J. S., Rebolo, R., Martín, E. L., \& Basri, G. 2000, ApJ, 524, 115

Zapatero Osorio, M. R., Béjar, V. J. S., Martín, E. L., et al. 2000, Sci, 290, 103

Zucker, S., \& Mazeh, T. 2000, ApJ, 531, L67

Zuckerman, B., \& Webb, R. A. 2000, ApJ, 535, 959 